\title{
EXTENDED SUPERGRAVITY WITH LOCAI SO(5) INVARIANCE
}

\author{
B. de Wit \\ NIKHEF-H - Amsterdam \\ and \\ H. Nicolai \\ CERN - Geneva
}

$\triangle B S T R A C T$

\begin{abstract}
We present a version of $\mathrm{N}=5$ supergravity'with local so(5) invariance and a lowest order calculation for $N=8$ supergravity with local $\mathrm{s}(8)$. The implications of these results and related aspects are discussed.
\end{abstract}





\section{INTRODUCTION}

Extended supergravity theories are invariant under $N$ independent local supersymmetries which are naturally combined with a rigid $S O(N)$ symmetry group [see, e.g., Ref. 1】. Already some time ago, it has been shown that this rigid invariance can be extended to a local one for $N=2$ and $3^{2}$ ). In the case of $N=4$, two different versions with local internal symmetry exist corresponding to the two variants of the $N=4$ theory 3 ), one with local $\mathrm{SU}(2) \times \mathrm{SU}(2) \quad 4)$ and another with local so(4) 5). In the presence of a gauged internal symmetry, the graded Poincaré algebra is replaced by the graded de Sitter algebra, and the invariant Lagrangian acquires a cosmological term.

Beyond $N=4$, no such results have been available so far. By consistent truncation, all extended supergravity models can be derived from the $N=8$ theory $\left.{ }^{6}\right)-8$ ). There are at least two reasons why theories with $N>4$ deserve even more attention than the case of lower $\mathbb{N}$ :

i) theories with higher $\mathbb{N}$, and in particular the $\mathbb{N}=8$ theory, have a better chance to be of phenomenological relevance. In fact, there have been some recent attempts in this direction ${ }^{9)}$. Therefore, it is quite important to study all aspects of these theories, such as the various possible ways in which higher $\mathbb{N}$ supergravity can be realized. Two generalizations of the original $\mathbb{N}=8$ theory have been discovered 10),11) which exhibit a cosmological term. But in both cases the internal symmetries remain of the rigid type.

ii) for $N>4$, extended supergravity theories with local $S O(N)$ exhibit interesting properties when they are quantized (under the assumption that these theories exist !). In the one-loop approximation, the cosmological constant which is induced by the gauging of $S O(N)$, is only finitely renormalized ${ }^{12}$ ). This is equally true for the gaugecoupling constant which, by supersymmetry, is directly related to the cosmological constant. This phenomenon may be viewed as another positive indication that supergravity will eventually lead to a consistent quantum theory of gravitation. Independent of the direct phenomenological applicability of these ideas, it is therefore of considerable theoretical interest to investigate higher $\mathbb{N}$ supergravities with gauged $S O(N)$.

In this paper we present $\mathbb{N}=5$ supergravity with a local so(5) invariance. The eull non--lincar structure of this theory is siven up to terms quartic in the spinor riedds. These latter terms are not required for 
a determination of the extra terms induced by the non-zero gauge-coupling and we have simply assumed that no inconsistencies arise in that sector. There is no problem of principle to complete these couplings, but under the previous assumption this would only amount to yet another check on the correctness of our results. At this point, we wish to remind the reader that the quartic terms for pure $N=8$ supergravity in five dimensions have been fully worked out by Cremmer 13).

The reason for considering the case of $N=5$ is twofold. First of al1, the construction of $N=5$ supergravity with gauged so(5) will constitute the first example of an extended supergravity theory to which the results of Ref. 12) apply and which has vanishing one-loop $\beta$ function. A second reason is that the case $\mathbb{N}=5$ is still algebraically manageable in contradistinction to the case $N \geq 6$; the spinless fields occur in the fundamental representation of $S O(5)$ which entails comparatively simple tensorial structures for the non-polynomial modifications present in these theories while, for $N \geq 6$, the scalar fields carry at least two indices, and one may build arbitrary strings.

We will also give the lowest order results for the case of $N=8$ with local $s O(8)$. These we compare with our direct $N=5$ calculation and establish full agreement up to the order we have investigated.

The plan of this paper is as follows : in Section 2 we present $N=5$ supergravity. We have constructed this theory by truncating the $I=8$ algebraic equations of Ref. 8) to $N=5$. Their solution leads directly to the $N=5$ Lagrangian and transformation rules. In addition, at the end of Section 2, we make contact with the work of Cremer and Julia by showing that our results are fully compatible with the coset formulation of $\mathbb{N}=5$ supergravity. The gauging of $S O(5)$ is described in section 3 where we also discuss the truncation to $N=4$. Section 4 contains oux conclusions, and, finally, we collect our lowest order $N=8$ results in an Appendix.

In our notation and conventions, we will mainly follow Ref. 8) and adopt the chiral notation used in that paper. Upper indices on spinors denote positive chirality, and raising (lowering) indices corresponds to complex conjugation [of., Eq. (2.1) or Ref. 8)]. The only difference is that, in this paper, the symbol $\left[i_{1}, \ldots, i_{n}\right]$ signifies antisymmetrization in the indices $i_{1}, \ldots, i_{n}$ with strength one. So, for instance, we define 


$$
\begin{aligned}
\delta_{j_{1}}^{i_{1}} \ldots i_{n} & \left.\equiv \delta_{j_{1}}^{\left[i_{1}\right.} \ldots \delta_{j_{n}}^{i_{n}}\right] \\
= & \frac{1}{n !}\left(\delta_{j_{1}}^{i_{1}} \ldots \delta_{j_{n}}^{i_{n}} \pm \text { permutations }\right)
\end{aligned}
$$

This accounts for some difference in numerical factors.

\section{EXTENDED SUPERGRAVITY WITH $N=5$}

The field content of $\mathbb{N}=5$ extended supergravity is as follows. The gravitational degrees of freedom are described by a vierbein field $V_{\mu}^{a}$ and a spin connection $\omega_{\mu}^{a b}$. There are three irreducible spinor fields which can be assigned to representations of chiral U(5): 5 gravitino fields $\psi_{\mu}^{i}$, 10 spin- $-\frac{1}{2}$ fields $x^{i j k}$ in the antisymmetric representation of $U(5)$ and a singlet spin- $\frac{1}{2}$ field $x^{678}$. Furthermore, the complex scalars $\phi^{i}=\left(\phi_{i}\right)^{*}$ belong to the fundamental representation of $U(5)$. The 10 Abelian vector fields $A_{\mu}^{i j}$ cannot transform under $U(5)$, and are assigned to the antisymmetric representation of the $S O(5)$ subgroup instead.

Following Ref. 8), the Lagrangian of $\mathbb{N}=5$ supergravity can be written as

$$
\begin{aligned}
& \mathscr{L}=-\frac{1}{2} V R(V, \omega)-\frac{1}{2} \varepsilon^{\mu \nu \rho \sigma} \bar{\psi}_{\mu}^{i} \gamma_{\nu} \vec{D}_{\rho} \psi_{\sigma i}- \\
& -\frac{1}{12} \vee \bar{\chi}^{i j k} \stackrel{\leftrightarrow}{\varnothing} x_{i j k}-\frac{1}{2} \vee \bar{\chi}^{678} \stackrel{\leftrightarrow}{D} x_{678}-\frac{1}{2} \vee g^{\mu v} a_{\mu}^{i} a_{v i}- \\
& -\frac{1}{8} V\left(\left(2 S^{i j, k l}-\delta^{i k} \delta^{j l}\right) F_{\mu v i j}^{+} F_{k l}^{+\mu v}+h . c .\right)- \\
& -\frac{1}{2} V\left(S^{i j, k \ell} F_{\mu v i j}^{+} O^{+\mu v k \ell}+h . c .\right)- \\
& -\frac{1}{12} V\left(\varepsilon^{i j k \ell m} \bar{\chi}_{i j k} \gamma^{\nu} \gamma^{\mu} \psi_{\nu e} a_{\mu m}+h . c .\right)- \\
& -\frac{1}{2} V_{\eta}\left(\bar{\chi}_{678} \gamma^{\nu} \gamma^{\mu} \psi_{\nu i} a_{\mu}^{i}+\text { h.c. }\right)+ \\
& +\frac{1}{4} \vee \bar{\chi}^{i j k} \gamma^{\mu} \chi_{i j e} B_{\mu k}^{e}-\frac{1}{2} \vee \bar{\chi}^{678} \gamma^{\mu} \chi_{678} B_{\mu k}^{k}+ \\
& +\frac{1}{2} \varepsilon^{\mu \nu \rho \sigma} \bar{\psi}_{\mu}^{i} \gamma_{v} \psi_{\sigma j} \beta_{\rho}^{j} i+ \\
& \text { t terms quartic in the spinor fields }
\end{aligned}
$$


$-4-$

In (2.1), and in the rest of this paper, we put the gravitational coupling constant equal to one. The Riemann curvature scalar is denoted by $R(V, \omega)$; $V$ is the vierbein determinant $V=$ aet $V_{\mu a}$. The self-dual and antiself-dual field strengths (with lower and upper indices, respectively) are defined by

$$
\begin{aligned}
& F+\mu v \equiv \frac{1}{2}\left(g^{\mu \rho} g^{v \sigma}+\frac{1}{2} V^{-1} \varepsilon^{\mu v \rho \sigma}\right)\left(\partial_{\rho} A_{\sigma}^{i j}-\partial_{\sigma} A_{\rho}^{i j}\right) \\
& F^{-\mu v i j} \equiv \frac{1}{2}\left(g^{\mu \rho} g^{v \sigma}-\frac{1}{2} V^{-1} \varepsilon^{\mu v \rho \sigma}\right)\left(\partial_{\rho} A_{\sigma}^{i j}-\partial_{\sigma} A_{\rho}^{i j}\right) \\
&\left(F^{+\mu v}{ }_{i j}\right)^{*}=F^{-\mu v i j}
\end{aligned}
$$

They enter the Lagrangian in a way which is not invariant under chiral U(5). The quantity $\mathrm{s}^{i j, k l}$ which depends in a non-polynomial fashion on the scalar fields $\phi^{i}$ also lacks manifest $U(5)$ invariance. It is symmetric in the antisymmetric index pairs $(i j)$ and $(k l)$, and is defined by the condition

$$
\left(\delta_{k e}^{i j}-\bar{S}_{i j, k e}\right) S^{k e, m n}=\delta_{m n}^{i j}
$$

where $\bar{S}$ is a $U(5)$ covariant object. By an appropriate choice of field variables, $\overline{\mathrm{s}}$ can be taken equal to

$$
\bar{S}^{i j, k e}=-\frac{1}{2} \varepsilon^{i j k e m} \phi_{m}
$$

The remaining substitutions that we have used ir (2.1) are manifestly U(5) covariant.

$$
\begin{aligned}
& O_{\mu v}^{+i j}=C_{k e}^{i j}\left\{-\frac{\sqrt{2}}{12} \eta \varepsilon^{k e m n p} \bar{\chi}_{m n p} \sigma_{\mu v} \chi_{678}-\right. \\
& \left.-\frac{1}{2} \bar{\psi}_{\lambda m} \sigma_{\mu v} \gamma^{\lambda} \chi^{k l m}+\frac{\sqrt{2}}{2} \bar{\psi}_{\rho}^{k} \gamma^{[\rho} \sigma_{\mu v} \gamma^{\sigma]} \psi_{\sigma}^{l}\right\} \\
& E_{k e}^{i j} C_{m n}^{k e}=\delta_{m n}^{i j} \\
& \left.E_{k e}^{i j}=e_{1} \delta_{k e}^{i j}+2 e_{2} \delta_{[k}^{[i} \phi^{j]} \phi_{l}\right]
\end{aligned}
$$




$$
C_{k e}^{i j}=\frac{1}{e_{1}} \delta_{k e}^{i j}-2 \frac{e_{2}}{e_{1}} \delta_{[k}^{[i} \phi^{j]} \phi_{l]}
$$

We have introduced here the scalar functions $e_{1}$ and $e_{2}$

$$
\begin{aligned}
& e_{1}=\left(1-|\phi|^{2}\right)^{-1 / 2},|\phi|^{2} \equiv \phi^{i} \phi_{i} \\
& e_{2}=\frac{1}{|\phi|^{2}}\left(1-\left(1-|\phi|^{2}\right)^{-1 / 2}\right)
\end{aligned}
$$

Observe that $e_{2}$ is regular at $\phi_{i}=0$. We also record the following useful relations

$$
\begin{aligned}
& |\phi|^{2} e_{2}=1-e_{1} \\
& e_{1}^{2}+e_{2}+e_{1} e_{2}=0
\end{aligned}
$$

The remaining quantities $a_{\mu}^{i}$ and $\mathcal{B}_{\mu j}^{i}$ are given by

$$
\begin{aligned}
& a_{\mu}^{i}=-\sqrt{2} e_{1}\left(\delta_{j}^{i}-e_{2} \phi^{i} \phi_{j}\right) \partial_{\mu} \phi^{j} \\
& B_{\mu j}^{i}=2 e_{2} \phi^{i} \overleftrightarrow{\partial}_{\mu} \phi_{j}+\left(\frac{1}{2} e_{1}^{2} \delta_{j}^{i}-e_{2}^{2} \phi^{i} \phi_{j}\right) \phi^{k} \overleftrightarrow{\partial}_{\mu} \phi_{k}
\end{aligned}
$$

where $\mathcal{B}_{\mu j}^{i}$ is anti-Hermitean, i.e., $\mathscr{B}_{\mu j}^{i}=-\mathcal{B}_{\mu j}{ }^{i}=-\left(\mathscr{B}_{\mu}^{j}{ }^{j}\right)^{*}$. In these equations we have kept the $S O(8)$ duality phase $\eta$ to make the truncation from $\mathrm{N}=8 \quad$ supergravity more explicit.

The results (2.1)-(2.14) were obtained by solving Eqs. (4.3)-(4.6) and $(4.13)-(4.18)$ of Ref. 8) for the various non-polynomial functions that occur in the $N=8$ theory. These equations were based on the conjectured $\mathrm{SU}(8)$ invariance of the field equations, and they should also be valid for the submultiplet that underlies the $N=5$ truncation. More precisely, repeating the analysis of Ref. 8) for $N=5$ would yisld the same equations, apart from an appropriate relabelling of indices. Therefore, the $\mathbb{N}=5$ equations can be written down directly, and owing to the simple representation content of the $\mathrm{N}=5$ scalars, they can be solved explicitly. 
The action of $\mathbb{N}=5$ supergravity is invariant under local supersymmetry transformations characterized by parameters $e^{i}$. Combining the results of Ref. 8) with the previous determination of the non-polynomial functions leads to the following transformation rules

$$
\begin{aligned}
& \delta V_{\mu}{ }^{a}=\bar{\varepsilon}^{i} \gamma^{a} \psi_{\mu i}+\text { h.c. } \\
& \delta \bar{\psi}_{\mu}^{i}=2 \bar{\varepsilon}^{i} \overleftarrow{D}_{\mu}+\bar{\varepsilon}^{j} B_{\mu}^{i} j+\frac{\sqrt{2}}{2} \bar{\varepsilon}_{j} \gamma_{\mu} \sigma \cdot \bar{F}-k e C_{k e}{ }^{i j}+ \\
& +\ldots * * \\
& \delta A_{\mu}^{i j}=\left(\bar{S}^{i j, k e}-\delta^{i k} \delta^{j e}\right) E_{k e}^{m n}\left(\bar{\varepsilon}^{p} \gamma_{\mu} \chi_{m n p}+2 \sqrt{2} \bar{\varepsilon}_{m} \Psi_{\mu n}\right) \\
& + \text { h.c. } \\
& \delta \bar{\chi}^{i j k}=\varepsilon^{i j k e m} \bar{\varepsilon}_{\ell} \gamma^{\mu} a_{\mu m}-3 \bar{\varepsilon}^{m} \sigma \cdot \bar{F}^{-r s} \delta_{m}^{[i} C_{r s}^{j k]}+ \\
& +\ldots \\
& \delta \bar{\chi}^{678}=-\eta \bar{\varepsilon}_{i} \gamma^{\mu} a_{\mu}^{i}+\ldots \\
& \delta \phi_{i}=-\frac{\sqrt{2}}{e_{1}}\left(\delta_{i}^{j}+\frac{e_{2}}{e_{1}} \phi_{i} \phi^{j}\right)\left(\eta \bar{\varepsilon}_{j} \chi_{678}+\frac{1}{6} \varepsilon_{j k e m n} \bar{\varepsilon}^{k} \chi^{l m n}\right)
\end{aligned}
$$

We have given the variations of the spinors only modulo terms that are quadratic in the Fermi fields. In (2.15), we have also introduced the U(5) covariant field strength

$$
\bar{F}_{\mu v i j}^{+}=S^{i j, k e}\left(F_{\mu v k e}^{+}+O_{\mu v}^{+} k e\right)
$$

Note that apart from the variations of the vector fields $A_{\mu}^{i j}$, the transformation rules (2.15) are manifestly $U(5)$ covariant.

An alternative approach to obtain $N=5$ supergravity is by consistent truncation of the complete $\mathbb{N}=8$ theory as it was constructed by Cremmer and Julia ${ }^{7)}$. For $\mathbb{N}=5$, their formulation is invariant under local $U(5)$ and

*) Remember $c_{k I}^{i j} \equiv\left(c_{i j}^{k I}\right)^{*}$. 
rigid SU $(5,1)$. An important aspect is that the scalars are then described as the coset $\mathrm{SU}(5,1) / \mathrm{U}(5)$. The $\mathrm{N}=5$ theory presented in this section would then be obtained, upon a suitable $U(5)$ gauge choice. Indeed our results are entirely consistent with these ideas, and the scalars $\phi^{i}$ can be viewed as corresponding to a particular parametrization of the coset space. $\mathrm{SU}(5,1) / \mathrm{U}(5)$. Namely, one finds the following coset representative 14$)$ in terms of $\phi^{i} \quad\left(|\phi|^{2} \leq 1\right)$

$$
\Sigma_{B}^{A}=\left[\begin{array}{c|c}
\delta_{j}^{i}-e_{2} \phi^{i} \phi_{j} & e_{1} \phi^{i} \\
\hline e_{1} \phi_{j} & e_{1}
\end{array}\right](A, B=1, \ldots, 6)
$$

Hence $\Sigma$ is an element of $S U(5,1)$, decomposed into blocks according to its compact $\mathrm{SU}(5)$ subgroup. The killing metric of $\mathrm{SU}(5,1)$ is $\eta_{\mathrm{AB}}=$ $=\operatorname{diag}(+++++)$, and we have

$$
\Sigma^{-1}=\eta \Sigma^{+} \eta, \operatorname{det} \Sigma=1
$$

To show the compatibility of $(2.17)$ with our results it suffices to calculate $\Sigma^{-1} \partial_{\mu} \Sigma$ which takes values in the Lie algebra of $S U(5,1)$. A straightforward computation yields

$$
\Sigma^{-1} \partial_{\mu} \Sigma=\left[\begin{array}{c|c}
\frac{1}{2} B_{\mu j}^{i}-\frac{1}{6} \delta^{i} j B_{\mu k}^{k} & -\frac{\sqrt{2}}{2} a_{\mu}^{i} \\
\hline-\frac{\sqrt{2}}{2} a_{\mu j} & \frac{1}{3} B_{\mu k}^{k}
\end{array}\right]
$$

The elements of the maximal compact subalgebra $U(5)$, which are entirely given in terms of $\mathcal{B}_{\mu}^{i}$, will play the role of the gauge fields of $U(5)$. This is indeed consistent with the way in which $\mathcal{B}_{\mu j}^{j}$ occurs in the Iagrangian and transformation rules. Hence, it is natural to introduce $U(5)$ covariant derivatives which allow us to re-express the non-diagonal blocks in (2.19) as 
$-8-$

$$
\left(D_{\mu} \Sigma \cdot \Sigma^{-1}\right)_{B}^{A}=-\frac{\sqrt{2}}{2}\left[\begin{array}{ll}
0 & a_{\mu}^{i} \\
a_{\mu j} & 0
\end{array}\right]
$$

with

$$
\begin{aligned}
& \left(\partial_{\mu} \Sigma\right)_{B}^{A} \equiv \partial_{\mu} \Sigma_{B}^{A}-\Sigma_{C}^{A} B_{\mu B}^{C} \\
& B_{\mu B}^{A} \equiv\left[\begin{array}{c|c}
\frac{1}{2} B_{\mu j}^{i}-\frac{1}{6} \delta_{j}^{i} B_{\mu k}^{k} & 0 \\
\hline 0 & \frac{1}{3} B_{\mu k}^{k}
\end{array}\right]
\end{aligned}
$$

The invariant Lagrangian for the scalars is then proportional to

$$
\mathscr{L}_{\text {scalar }} \sim \operatorname{Tr}\left(\partial_{\mu} \Sigma \partial_{\mu} \Sigma^{-1}\right)=-\operatorname{Tr}\left(\partial_{\mu} \Sigma \cdot \Sigma^{-1}\right)^{2}
$$

which, by Eq. (2.20), is identical to the corresponding term in Eq. (2.1).

These observations complete our discussion of extended supergravity with rigid SO $(5)$.

3. $N=5$ SUPERGRAVITY WITH LOCAL SO (N)

The extension of the rigid $S O(5)$ invariance of $N=5$ supergravity to a local gauge group follows the same pattern as the treatment for lower Nos. Namely, one first makes the derivatives and the field strengths covariant with respect to local $S O(5)$ using the vector fields $A_{\mu}^{i j}$ as $S O(5)$ gauge fields. That is, we perform the substitutions

$$
\begin{aligned}
& \partial_{\mu} T_{j_{1} \ldots j_{n}} \longrightarrow \partial_{\mu} T_{j_{1} \ldots j_{n}} \equiv \\
& \equiv \partial_{\mu} T_{j_{1} \ldots j_{n}}-g A_{\mu}^{j_{1} k} T_{k j_{2} \ldots j_{n}}-\ldots-g A_{\mu}^{j_{n k}} T_{j_{1} \ldots j_{n-1} k}
\end{aligned}
$$


on all so(5) tensors $\mathbb{T}_{j_{1}} \ldots j_{n}$, and

$$
\begin{aligned}
F_{\mu v}{ }^{i j} & =2 \partial_{[\mu} A_{v]}^{i j} \rightarrow \\
\rightarrow F_{\mu v} i j & =2 \partial_{[\mu} A_{\nu]}^{i j}-2 g A_{[\mu}^{i k} A_{\nu]}{ }^{k j}
\end{aligned}
$$

All fields, except $A_{\mu}^{i j}$, have been assigned to $S U(5)$ representations, but since we gauge only the $S O(5)$ subgroup, the $S U(5)$ invariance of the field equations will be violated for finite gauge-coupling constant $g$.

When establishing the supersymmetry invariance of the modified action, the commutator of two covariant derivatives leads to a new $g$ dependent variation through the Ricci identity

$$
\left[D_{\mu}, D_{\nu}\right]=-g F_{\mu \nu}
$$

To cancel the effect of $(3.3)$, one must introduce new terms in the Lagrangian and transformation rules, in complete analogy with lower $N$ calculations 2$), 4), 5)$. These terms in turn give rise to new variations which no longer contain the field strengths, and the requirement of supersymmetry then leads to a unique determination of all extra terms. Since a laxge number of independent variations must cancel in this way, it is by no means clear that the result of such a calculation is internally consistent. But for $N=5$, this approach turns out to be successful. We have determined all modifications to Lagrangian and transformation rules, and established the invariance of the action for all variations, except those involving quartic spinorial terms. Let us now summarize our results.

We parametrize the $g$ dependent modifications in the following way

$$
\begin{aligned}
& \delta_{g} \bar{\Psi}_{\mu}^{i}=-\sqrt{2} g \bar{\varepsilon}_{j} \gamma_{\mu} A_{1}^{j i} \\
& \delta_{g} \bar{x}^{i j k}=-2 g \bar{\varepsilon}^{l} A_{2 l}{ }^{i j k} \\
& \delta_{g} \bar{\chi}^{678}=-2 g \eta \bar{\varepsilon}^{i} A_{4 i} \\
& \mathscr{L}_{g}=\mathscr{L}_{g}^{(1)}+\mathscr{L}_{g}^{(2)}+\mathscr{L}_{g}^{(3)}+\mathcal{L}_{g}^{(4)}
\end{aligned}
$$


$-10-$

with

$$
\begin{aligned}
V^{-1} \mathscr{L}_{g}^{(1)}= & g^{2}\left[2+4 e_{1}^{2}-\frac{1}{2} e_{1}^{4}\left(|\phi|^{4}-\left(\phi_{i}\right)^{2}\left(\phi^{j}\right)^{2}\right)\right] \\
V^{-1} \mathcal{L}_{g}^{(2)}= & \sqrt{2} g A_{1}^{i j} \bar{\psi}_{\mu i} \sigma^{\mu v} \psi_{v j}+h . c . \\
V^{-1} \mathcal{L}_{g}^{(3)}= & \frac{1}{6} g A_{2 i}{ }^{j k e} \bar{\psi}_{\mu}^{i} \gamma^{\mu} \chi_{j k e}+h . c .+ \\
& +g \eta A_{4}^{i} \bar{\psi}_{i}^{\mu} \gamma_{\mu} \chi^{678}+\text { hic. } \\
V^{-1} \mathscr{L}_{g}^{(4)}= & g A_{3}^{i j k, \operatorname{lmn}} \bar{\chi}_{i j k} \chi_{e m n}+\text { hic. }+ \\
+ & g \eta A_{5} \varepsilon^{i j k e m} \phi_{e} \phi^{m} \bar{\chi}_{i j k} \chi_{678}+\text { hic. }
\end{aligned}
$$

Here we have used the definitions $|\phi|^{2} \equiv \phi^{i} \phi_{i}$ and $\left(\phi_{i}\right)^{2} \equiv \phi_{i} \phi_{i}$. Notice that the Lagrangian does not contain terms quadratic in $x^{678}$. The quantities $A_{1-5}$ depend on the scalar fields $\phi^{i}$ and are given by

$$
A_{1}^{i j}=e_{1} \delta^{i j}+\frac{1}{2} e_{2}^{2}\left(|\phi|^{2} \phi^{i} \phi_{j}+|\phi|^{2} \phi_{i} \phi^{j}-2\left(\phi_{n}\right)^{2} \phi^{i} \phi^{j}\right)(3.12)
$$

$$
\begin{gathered}
A_{2 e} e^{i j k}=e_{1} \varepsilon^{i j k l m} \phi_{m}-e_{1} e_{2} \varepsilon^{i j k m n} \phi_{m} \phi^{n} \phi_{l}+ \\
+3 e_{1}^{2} \delta_{l m n}^{i j k} \phi_{m} \phi^{n}
\end{gathered}
$$

$$
A_{3}^{i j k, \operatorname{lmn}}=\frac{\sqrt{2}}{288} \varepsilon^{i j k p q} \varepsilon^{\text {lemurs }} \varepsilon_{p q r s t} A_{4}^{t}
$$

$$
A_{4}^{i}=-e_{1}^{2} \phi_{i}-e_{1} e_{2}\left(\phi_{j}\right)^{2} \phi^{i}
$$




$$
A_{5}=-\frac{\sqrt{2}}{4} e_{1}^{2}
$$

Observe that our notation is such that the functions $A_{1-5}$ are treated as if they were SU(5) covariant, although their explicit expressions are only covariant with respect to SO(5)。

We now briefly compare our results with those of gauged so(4) supergravity which also contains the cases of $N \leq 3$. In this truncation, we have $(i, j, k, l=1, \ldots, 4)$

$$
\begin{aligned}
& \phi_{5} \equiv \phi, \quad \phi_{1}=\ldots=\phi_{4}=0 \\
& e_{1}=\left(1-|\phi|^{2}\right)^{-1 / 2}
\end{aligned}
$$

$$
A_{1}^{i j}=e_{1} \delta^{i j}, A_{2 e}{ }^{i j k}=e_{1} \varepsilon^{i j k e} \phi
$$

$$
A_{3}^{i j k, \ln n}=0
$$

and also

$$
\psi_{\mu}^{5}=x^{678}=x^{i j 5}=0
$$

This leads to a considerable simplification in the formulas (3.8)-(3.11) because all non-trivial tensorial structures disappear for $\mathbb{N} \leq 4$ and it is in agreement with the results of Ref. 5). In particular, we note that rigid $\mathrm{U}(1)$ invariance in $\mathrm{A}_{2}$ is restored in the $\mathrm{N}=4$ truncation.

\section{CONCLUSIONS}

In this paper we have demonstrated that the $\mathbb{N}=5$ supergravity with local $50(5)$ exists. The exciting implication of this result is that there is now at least one example to which the considerations of Ref. 12) apply : the cosmological constant and thus the gauge coupling constant are only finitely renormalized at one $100 \mathrm{p}$ in this theory. The construction of gauged $N=5$ supergravity lends credibility to the results of Ref. 12) which would have otherwise consisted of vacuous statements. Our results also show that there is no borderline which qualitatively separates the cases $\mathbb{N} \leq 4$ and $\mathrm{N}>4$. 
In view of our construction, one might wonder whether extended supergravity theories with local so(N) also exist for $\mathbb{N}=6,7,8$. There is no group theoretic argument against such a construction since the corresponding graded de sitter algebras are known to exist ${ }^{15)}$. We have, in fact, attempted to gauge $s o(8)$ in the maximally extended $\mathbb{N}=8$ theory. So far, howevex, our results are incomplete being based on the partial construction of Ref. 6), and we have therefore relegated them to an Appendix. For a full construction, a knowledge of the complete $\mathbb{N}=8$ theory in its manifestly $\mathrm{SO}(8)$ invariant form is an obvious prerequisite.

There also remain a few more or less exotic possibilities to be explored. One is that one might try to gauge a subgroup of $\mathrm{SO}(\mathrm{N})$. That this idea is not as outlandish as it may seem at first glance is evident from the existence of the gauged $N=4$ theory in the version with local $\mathrm{SU}(2) \times \mathrm{SU}(2)$ where one has two independent coupling constants ${ }^{4)}$. However, the case of $N=4$ is special not only in that the covering group of $\mathrm{SO}(4)$ is $\mathrm{SU}(2) \times \mathrm{SU}(2)$ but also in that, surprisingly, there is no rigid graded de Sitter algebra for SU(2)x $\times \mathrm{SU}(2)$ 16). For $\mathbb{N}=8$, another possibility is to try to gauge the $\mathrm{s}(7)$ symmetry in that version of the $N=8$ theory that is obtained by direct dimensional reduction from 11-dimensional supergravity 17$), *$ ) in which some spinless degrees of freedom are represented by antisymmetric tensors. Yet, the problem of minimal coupling inconsistencies seems hard to avoid in this case.

At any rate, further studies are clearly necessary, and we believe that more structure remains to be unravelled for $\mathbb{N} \geq 6$. The intriguing quantum properties of $\mathbb{N} \geq 5$ models should be regarded as yet an indication that supergravity offers a promising approach to the construction of a consistent quantum theory of gravitation.

*) This suggestion is due to E. Cremmer (P. Townsend, private communication). Note that the covering group of $S O(7)$ is the spin 7 group which has an eight-dimensional fundamental representation to which the supersymmetry generators and the gravitino fields are assigned. 
$-13-$

APPENDIX - AN ATTEMPT AT GAUGING ȘO(8)

The extension of the rigid $S O(8)$ invariance of $N=8$ supergravity to a local $s o(8)$ invariance is completely analogous to the procedure outlined in section 3. We here simply collect our lowest order results; for the pure $\mathbb{N}=8$ Lagrangian and transformation rules, we refer the reader to Ref. 6) on which our calculations are based. We also reinsert appropriate powers of $x$ to make more explicit which order of $x$ we are dealing with.

$$
\begin{aligned}
& \text { We have found the following } g \text { dependent new variations }(i, j, \ldots= \\
& =1, \ldots ., 8) \\
& \delta_{g} \bar{\psi}_{\mu}^{i}=-\sqrt{2} \frac{g}{k^{2}} \bar{\varepsilon}_{j} \gamma_{\mu} A_{1}^{j i} \\
& \delta_{g} \bar{x}^{i j k}=-2 \frac{g}{k} \bar{\varepsilon}^{e} A_{2 e}{ }^{i j k} \\
& A_{1}^{i j}=e_{1} \delta^{i j}+\frac{\sqrt{2}}{96} k^{3}\left(\phi^{i k m n} \phi_{m n p q} \phi^{p q k j}+h \cdot c\right)+(A .3) \\
& +O\left(k^{4}\right)
\end{aligned}
$$

$A_{2 e}{ }^{i j k}=-\frac{\sqrt{2}}{2} e_{1} \phi^{i j k e}-\frac{3}{8} k \delta_{n p q}^{i j k} \phi_{e n r s} \phi^{r s p q}+O\left(k^{2}\right)$ (A.4)

Furthermore

$$
\mathscr{L}_{g}=\mathscr{L}_{g}^{(1)}+\mathcal{L}_{g}^{(2)}+\mathcal{L}_{g}^{(3)}+\mathscr{L}_{g}^{(4)}
$$

where

$$
\begin{aligned}
& V^{-1} \mathcal{L}_{g}^{(1)}=\frac{g^{2}}{k^{4}}\left(2+4 e_{1}^{2}\right)+O\left(k^{0}\right) \\
& V^{-1} \mathcal{L}_{g}^{(2)}=\sqrt{2} \frac{g}{k} A_{1 i j} \bar{\psi}_{\mu}^{i} \sigma^{\mu v} \psi_{v}^{j}+h . c .
\end{aligned}
$$

*) Using the identities in the Appendix of Ref. 8), one can show that
cubic term in (A.3) is proportional to $\delta^{i j}$ and vanishes in the $\mathrm{N}=5$ truncation. 


$$
\begin{aligned}
& V^{-1} \mathscr{L}_{g}^{(3)}=\frac{1}{6} g A_{2}^{i} j k e \bar{\psi}_{i}^{\mu} \gamma_{\mu} \chi^{j k l}+h \cdot c . \\
& V^{-1} \mathscr{L}_{g}^{(4)}=g A_{3}^{i j k, \operatorname{lm} n} \bar{\chi}_{i j k} \chi_{e m n}+h . c .
\end{aligned}
$$

with

$$
\begin{aligned}
A_{3 i j k, l m n}=\frac{1}{8} \delta_{i j k}^{p q r} \delta_{l m n}^{s t u}\left(\phi_{p q s t} \delta_{r u}-\frac{\sqrt{2}}{12} k \phi_{p q r v} \phi_{s t u v}+\right. \\
\left.+\frac{3 \sqrt{2}}{12} k \phi_{p q u v} \phi_{s t r v}\right)+O\left(k^{2}\right)
\end{aligned}
$$

The function $e_{1}$ here corresponds to the one that has been introduced in $(2.9)$

$$
e_{1} \equiv\left(1-\frac{k^{2}}{96} \phi^{i j k e} \phi_{i j k e}\right)^{-1 / 2}
$$

As for our notation, the comments after Eq. (3.16) are also valid here. Observe also that the scalar field potential may be represented as the sum of two terms proportional to $A_{1 i j} A_{1}^{i j}$ and $A_{2 j k I}^{i} A_{2 i}^{j k l}$, respectively, as in the
case $N=5$.

We have now established the invariance of the action with respect to the above modifications for the following terms : $g \bar{\epsilon} \psi F_{\mu \nu}$ and $g \bar{\varepsilon} \times F_{\mu \nu}$ terms through order $x, g \bar{\epsilon} \psi \mathbb{D}_{\mu} \phi$ terms through order $x, g \bar{\varepsilon} \times D_{\mu} \phi$ terms through order $x^{0}$, and $g^{2} \bar{\varepsilon} \psi$ through $O\left(x^{0}\right)$ and $\bar{g}^{2} \bar{\varepsilon} \times$ terms through order

The $N=5$ truncation is obtained as follows 6$), 7$ ): we keep $v_{\mu a}$; $\psi_{\mu}^{i} ; A_{\mu}^{i j} ; x^{i j k}, x^{678}$ and $\phi_{i 678} \equiv-\sqrt{2 \eta \phi_{i}}$ as well as $\phi_{i j k l}=-\sqrt{2 \varepsilon_{i j k l m}} \phi^{m}$ $(i, j, \ldots=1, \ldots, 5)$. There is a direct correspondence between $A_{1-3}(\mathrm{~N}=8)$ and $A_{1-3}(N=5)$, whereas the functions $A_{4,5}(N=5)$ oxiginate from the $N=8$ results for $A_{2}$ and $A_{3}$, respectively. In this way, each term in (3.4)(3.11) has its direct counterpart in Eqs. (A.1)-(A.9), and we have verified up to the available orders in $x$ that there is complete agreement. Hence, we have a powerful check on our $N=8$ calculations. 
We also note the amusing fact that the $N=5$ truncations of the second and third terms in (A.10) vanish in the $\bar{x}_{i j k} x_{i j k}$ and $\bar{x}_{i j k} x_{678}$ sectors; they only contribute in the $\bar{x}_{i j k} x_{678}$ sector which is again in agreement with the results of Section 3 .

It is clear that we have some evidence that a gauging of so(8) in the $N=8$ theory is possible although an obstruction could arise in highex orders of $x$. However, our complete $\mathbb{N}=5$ results impose the non-trivial constraint that such an inconsistency must vanish at least in the $N=5$ truncation. 


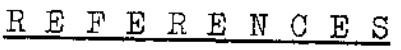

1) J. Scherk, in Recent Developments in Gravitation, Summer Institute, Cargèse (1978), Eds. M. Lévy and S. Desex (Plenum Press, New York, 1979);

P. van Nieuwenhuizen, Phys. Reports, in press.

2) D.Z. Freedman and A. Das, Nuclear Phys. B120 (1977) 221;

E.S. Fradkin and M.A. Vasiliev, Lebedev Institute preprint (1976).

3) A. Das, Phys.Rev. D15 (1977) 2805;

E. Cremmer, J. Scherk and S. Ferrara, Phys.Letters 68B (1977) 234;

E. Cremmer and J. Scherk, Nuclear Phys. B127 (1977) 259;

E. Cremmer, J. Scherk and S. Ferrara, Phys. Letters 74B (1978) 61.

4) D.Z. Freedman and J.H. Schwarz, Nuclear Phys. B137 (1978) 333.

5) E. Cremmex, Jo Scherk and S. Ferrara, unpublished.

6) B. de Wit and D.Z. Freedman, Nuclear Phys。B130 (1977) 105.

7) E. Cremmer and B. Julia, Phys.Letters 80B (1978) 48; Nuclear Phys.
B159 (1979) 141.

8) B. de Wit, Nuclear Phys. B158 (1979) 189.

9) T.I. Curtright and P.G.O. Freuna, in Supergravity, Eds. P. van Nieuvenhuizen and D.Z. Freedman, North Holland Publ.Co. (1979).

J. Ellis, M.K. Gaillard and B. Zumino, Phys.Letters 94B (1980) 343;

J. Ellis, M.K. Gaillard, I. Maiani and B. Zumino, in Proceedings Europhysics Study Conference on Unification of Fundamental Interactions, Erice (1980)
(Plenum Press, 1981);

J.P. Derendinger, S. Ferrara and C.A. Savoy, CERN preprint TH. 3025 (1981).

10) E. Cremmer, J. Scherk and J.H. Schwarz, Phys.Letters 84B (1979) 83.

11) A. Aurilia, H. Nicolai and P.K. Townsend, Nuclear Phys. B176 (1980). 509.

12) S.M. Christensen, M.J. Duff, G.W. Gibbons and M. Rocek, Phys.Rev. Letters
45 (1980) 161.

13) E. Cremmer, in Proceedings of the Nuffield Supergravity Workshop (1980), Eds. S.W. Hawking and M. Rocek (Cambridge University Press - in press).

14) R. Gilmore, "Iie Groups, Lie Algebras and Some of Their Applications",
Wiley, New York (1974).

15) P.G.O. Freund and J. Kaplansky, J.Math.Phys. 17 (1976) 228;

W. Nahm, V. Rittenberg and M. Scheunert, Phys.Letters $51 \mathrm{~B}$ (1976) 383.

16) J. Wess and $B_{0}$ Zumino, unpublished.

17) E. Cremmer, B. Julia and J. Scherk, Phys.Jetters 76B (1978) 409. 